\title{
Regulation of Blood Coagulation Factors XI and XII in Patients with Acute and Chronic Cerebrovascular Disease: A Case-Control Study
}

\author{
Peter Kraft ${ }^{\mathrm{a}, \mathrm{d}} \quad$ Christiane Drechsler $^{\mathrm{b}} \quad$ Ignaz Gunreben ${ }^{\mathrm{a}} \quad$ Peter Ulrich Heuschmann ${ }^{\mathrm{c}} \mathrm{d}$ \\ Christoph Kleinschnitz ${ }^{a}$
}

Departments of ${ }^{a}$ Neurology, ${ }^{b}$ Internal Medicine and ${ }^{c}$ Clinical Trial Center, University Hospital Würzburg, Würzburg, ${ }^{d}$ Institute of Clinical Epidemiology and Biometry, Comprehensive Heart Failure Center, University of Würzburg, Würzburg, Germany

\section{Key Words}

Biomarker · Factor XI · Factor XII · Ischemic stroke · Chronic cerebrovascular disease

\begin{abstract}
Background: Animal models have implicated an integral role for coagulation factors XI (FXI) and XII (FXII) in thrombus formation and propagation of ischemic stroke (IS). However, it is unknown if these molecules contribute to IS pathophysiology in humans, and might be of use as biomarkers for IS risk and severity. This study aimed to identify predictors of altered FXI and FXII levels and to determine whether there are differences in the levels of these coagulation factors between acute cerebrovascular events and chronic cerebrovascular disease (CCD). Methods: In this case-control study, 116 patients with acute ischemic stroke (AIS) or transitory ischemic attack (TIA), 117 patients with CCD, and 104 healthy volunteers (HVs) were enrolled between 2010 and 2013 at our University hospital. Blood sampling was undertaken once in the CCD and HV groups and on days 0,1 , and 3 after stroke onset in patients with AIS or TIA. Correlations between serum FXI and FXII levels and demographic and clini-
\end{abstract}

cal parameters were tested by linear regression and analysis of variance. Results: The mean age of AIS/TIA patients was $70 \pm 12$. Baseline clinical severity measured with NIHSS and Barthel Index was $4.8 \pm 6.0$ and $74 \pm 30$, respectively. More than half of the patients had an AIS (58\%). FXI levels were significantly correlated with different leukocyte subsets $(p<$ 0.05). In contrast, FXII serum levels showed no significant correlation $(p>0.1)$. Neither FXI nor FXII levels correlated with CRP ( $p>0.2)$. FXII levels were significantly higher in patients with CCD compared with those with AIS/TIA (mean \pm SD $106 \pm 26 \%$ vs. $97 \pm 24 \%$; univariate analysis: $p<0.05$ ); these differences did not reach significance in multivariate analysis adjusted for sex and age. FXI levels did not differ significantly between study groups. Sex and age were significantly associated with FXI and/or FXII levels in patients with AIS/TIA ( $p<0.05)$. In contrast, no statistical significant influence was found for treatment modality (thrombolysis or not), pre-treatment with platelet inhibitors, and severity of stroke. Conclusions: In this study, there was no differential regulation of FXI and FXII levels between disease subtypes but biomarker levels were associated with patient and clinical characteristics. FXI and FXII levels might be no valid biomarker for predicting stroke risk.

(c) 2014 S. Karger AG, Base

\section{KARGER}

E-Mail karger@karger.com

www.karger.com/ced
(C) 2014 S. Karger AG, Basel

$1015-9770 / 14 / 0385-0337 \$ 39.50 / 0$
Dr. Christoph Kleinschnitz

Department of Neurology, University Hospital Würzburg Josef-Schneider Strasse 11

DE-97080 Würzburg (Germany)

E-Mail christoph.kleinschnitz@uni-wuerzburg.de 


\section{Introduction}

The fundamental involvement of coagulation mechanisms in lesion development after ischemic stroke (IS) is well recognized $[1,2]$. However, the specific pathophysiologic role of plasma coagulation factors and platelets and their interaction with endothelial cells or other resident cell types in IS have not been fully elucidated. Research advances in transgenic mouse models and specific antibodies have facilitated the development of animal models of IS, which, in turn, have led to the delineation of pathways responsible for pathologic thrombus formation [2, 3]. In such preclinical stroke models, major pathophysiologic roles in arterial thrombus formation were identified for factor XIIa (FXIIa) and its main substrate, factor XI (FXI) $[4,5]$.

Factor XII (FXII) and FXI form the essential components of the coagulation cascade, which consists of several serially connected serine proteases within two distinct pathways (the extrinsic and intrinsic pathway) [6]. Activation of the coagulation cascade prompts a sequence of reactions that lead to thrombin generation and ultimately to fibrin formation, thereby stabilizing growing platelet thrombi. While it is well established that the extrinsic coagulation pathway is triggered by tissue factor at sites of vascular damage, the physiologic trigger for the intrinsic coagulation cascade remains elusive [2]. Seminal in vivo studies have shown that negatively charged ribonucleic acid or polyphosphates are potent activators of FXII $[7,8]$. In addition to a role in the intrinsic part of the coagulation cascade, FXII is also the starting point for the kallikrein-kinin system (which is linked to hallmark features of IS, such as edema formation, inflammation, and thrombosis) [9], thereby merging thrombus formation and inflammation in a process called 'thrombo-inflammation' [10, 32].

Several experimental studies have reported that blocking FXI $[11,12]$ as well as FXII $[4,13]$ leads to reduced ischemic neurodegeneration in rodent stroke models. Development of the highly selective FXII inhibitor, recombinant human albumin-tagged infestin- 4 enabled verification of the results from transgenic animal models [5, 14], and supported the translational impact of this novel therapeutic approach. Most importantly, targeting FXI and FXII provided its beneficial effects in IS models without a concomitant increase in bleeding complications, thus providing a basis for the unique and intriguing concept of a potential 'bleeding-free' antithrombotic approach [2].

Increasing clinical evidence lends support to a causal association between FXI [15-18] or FXII $[19,20]$ levels and acute stroke risk and functional outcome after IS. Moreover, the available evidence points toward a Ushaped correlation between FXIIa levels and disease independent overall survival [21]. Nevertheless, many questions remain unanswered. For instance, the processes involved in the regulation of FXI and FXII expression during IS await clarification, as does the relationship between FXI or FXII levels and genetic polymorphisms [22], demographic features [23, 24], and therapeutic interventions [25], among others. Additionally, despite recent experimental evidence that FXII regulates the pathologic process of thrombus formation on ruptured plaques [26], to our knowledge, there appears to be lack of published data on the regulation of FXI and FXII in patients with chronic cerebrovascular disease (CCD).

This case-control study aimed to identify demographic and clinical factors being associated with FXI and FXII serum levels, and to evaluate whether levels of these coagulation factors differ between patients with acute cerebrovascular events and CCD.

\section{Methods}

\section{Data Collection}

Patients with acute cerebrovascular events (acute ischemic stroke [AIS], transitory ischemic attack [TIA]) and CCD were included in the study. Healthy volunteers (HV) from the local population were selected as controls. Inclusion criteria were blood withdrawal within $24 \mathrm{~h}$ after symptom onset in patients with AIS (defined as acute ischemic lesion on brain imaging) and TIA (no acute lesion); presentation with extracranial and/or intracranial stenosis of the large cerebral arteries with $(n=66)$ or without $(n=51)$ a history of AIS or TIA for the CCD group; and no history of stroke, myocardial infarction, or peripheral arterial disease for the HV group. Hemorrhagic stroke, age $<18$ years, and known platelet dysfunction or plasmatic coagulation disorders based on a detailed medical history led to exclusion from the study. Consecutive patients were recruited from the Stroke Unit (inpatients diagnosed with TIA or AIS), the outpatient department for CCD, or from a convenient sample of the local population (HV) that responded to a request by posters at the Neurology Department, University Hospital of Würzburg, Germany, between September 2010 and January 2013. Informed written consent was obtained from all participants. The study protocol was approved by the ethics committee of the medical faculty of the University of Würzburg, Germany (reference number 65/2010). Study participation had no influence on treatment and patient care. Overall, 116 patients with AIS or TIA, 117 patients with CCD, and $104 \mathrm{HV}$ fulfilled the inclusion criteria and took part in the study.

In patients with AIS or TIA, TOAST (Trial of Org 10172 in Acute Stroke Treatment) criteria [27] were applied in an adapted form: (1) cardioembolism; (2) large-artery atherosclerosis; (3) small-vessel occlusion; or (4) other determined or undetermined etiology. In addition, the National Institute of Health Stroke Scale 
(NIHSS) [28] and Barthel Index score [29] were documented on patient admission. For each patient, data on the interval between symptom onset and blood withdrawal, platelet inhibitor pretreatment, and acute stroke therapy modality (thrombolysis vs. no thrombolysis) were documented.

\section{Blood Collection and Measurements}

Blood sampling was performed on day 0,1 , and 3 in patients with acute cerebrovascular disease, and once in patients with CCD and in HV between 08.00 and 12.00 hours, from an antecubital vein using a 21 -gauge butterfly needle. Preanalytic preparations for blood collection followed a specific standard operating procedure. Only non-hemolyzed blood samples were analyzed. FXI, FXII, differential hematology parameters, and C-reactive protein (CRP, only in the AIS/TIA group) were analyzed at the Division of Laboratory Medicine of the University Hospital Würzburg.

\section{Statistical Analysis}

Continuous variables are expressed as a mean value with standard deviation or median with interquartile range, as appropriate. Categorical variables are expressed as percentages. The association between FXI or FXII levels and a range of demographic and clinical characteristics was explored: age, sex, neurologic scales, disease modality (TIA or AIS), TOAST criteria, duration between symptom onset and blood withdrawal, NIHSS, Barthel Index, treatment modality (intravenous thrombolysis or not), and intake of platelet inhibitors in the days before blood withdrawal. The $p$ values for comparisons across groups of clinical and demographic characteristics were derived from analysis of variance (ANOVA), and the chi-square test, as appropriate. In order to identify potential predictors of FXI and FXII levels, a linear regression model was applied that included all variables without collinearity in a multivariate model with adjustment for age and sex. Coefficients and corresponding 95\% confidence intervals were estimated using the model. FXI and FXII levels were compared between the different patients groups (AIS/TIA inpatients, CCD outpatients, or HV), and distribution analyzed using the Kolmogorov-Smirnov test. Levels of FXI and FXII were assumed to take a normal distribution and were compared using ANOVA with a Bonferroni post-hoc test and additionally adjusted for age and sex. All reported p values are two-sided and a $\mathrm{p}$ value $<0.05$ was considered statistically significant. Analyses were performed using SPSS Version 21 and SAS software version 9.1 (SAS Institute Inc., Cary, N.C., USA).

\section{Results}

\section{Descriptive Analysis of the Patients with an Acute Cerebrovascular Event}

A total of 116 patients with AIS/TIA were included in this study. The mean age of these patients was $70 \pm 12$; $53 \%$ of the total number were male. The baseline clinical severity measured with NIHSS and Barthel Index was $4.8 \pm 6.0$ and $74 \pm 30$, respectively. More than half of the patients had an AIS (58\%). The demographic and clinical characteristics of the patients with acute cerebrovascular events are summarized in table 1.

Regulation of FXI and FXII in

Cerebrovascular Disease
Table 1. Baseline characteristics of acute ischemic stroke (AIS)/ transitory ischemic attack (TIA) patients

\begin{tabular}{|c|c|}
\hline Characteristic & $\begin{array}{l}\text { Value } \\
(\mathrm{n}=116)\end{array}$ \\
\hline Age, years & $70 \pm 12$ \\
\hline \multicolumn{2}{|l|}{ Sex, n (\%) } \\
\hline Male & $62(53)$ \\
\hline Female & $54(47)$ \\
\hline \multicolumn{2}{|l|}{ Modality, n (\%) } \\
\hline AIS & $67(58)$ \\
\hline TIA & $49(42)$ \\
\hline \multicolumn{2}{|l|}{ TOAST criteria, n (\%) } \\
\hline Cardioembolism & $70(60)$ \\
\hline Large-artery atherosclerosis & $4(3)$ \\
\hline Small-vessel occlusion & $12(10)$ \\
\hline Other determined or undetermined etiology & $30(26)$ \\
\hline Thrombolysis, n (\%) & $34(29)$ \\
\hline \multicolumn{2}{|l|}{ Comorbidities, n (\%) } \\
\hline Hypertension & $105(91)$ \\
\hline Diabetes mellitus & $41(35)$ \\
\hline Hyperlipidemia & $80(69)$ \\
\hline Renal failure & $10(9)$ \\
\hline Atrial fibrillation & $37(32)$ \\
\hline Persistent foramen ovale & $28(24)$ \\
\hline Heart failure & $5(4)$ \\
\hline Coronary artery disease & $8(7)$ \\
\hline Family history of stroke & $11(9)$ \\
\hline Smoking, n (\%) & $18(16)$ \\
\hline Platelet inhibitor before blood withdrawal, n (\%) & $87(75)$ \\
\hline Anticoagulation before blood withdrawal, n (\%) & $8(7)$ \\
\hline Lipid-lowering drug before blood withdrawal, n (\%) & $36(31)$ \\
\hline \multicolumn{2}{|l|}{ National Institutes of Health Stroke Scale at } \\
\hline Barthel index at admission & $74 \pm 30$ \\
\hline Body mass index, $\mathrm{kg} / \mathrm{m}^{2}$ & $27 \pm 5$ \\
\hline $\mathrm{HbA}_{1 \mathrm{c}}, \mathrm{mmol} / \mathrm{mol}$ hemoglobin & $46 \pm 13$ \\
\hline \multicolumn{2}{|l|}{ Lipid profile, $\mathrm{mmol} / \mathrm{l}$} \\
\hline Total cholesterol & $202 \pm 52$ \\
\hline Low-density lipoprotein & $121 \pm 45$ \\
\hline High-density lipoprotein & $51 \pm 15$ \\
\hline Triglycerides & $157 \pm 153$ \\
\hline $\begin{array}{l}\text { Duration between symptom onset and blood } \\
\text { withdrawal, } \mathrm{h}\end{array}$ & $14 \pm 7$ \\
\hline
\end{tabular}

$\mathrm{HbA}_{1 \mathrm{c}}=$ Glycated hemoglobin; TOAST $=$ Trial of Org 10172 in Acute Stroke Treatment.

\section{Comparison of FXI and FXII Levels in AIS/TIA}

Patients, CCD Patients, and HV

FXI and FXII levels by patient subtype (acute cerebrovascular events [AIS or TIA] or CCD) were compared with $\mathrm{HV}$. While there were no significant differences in FXI levels $(\mathrm{p}=0.26)$, FXII levels showed a significant difference between the AIS/TIA $(97 \pm 24 \%)$ and CCD (106 \pm 


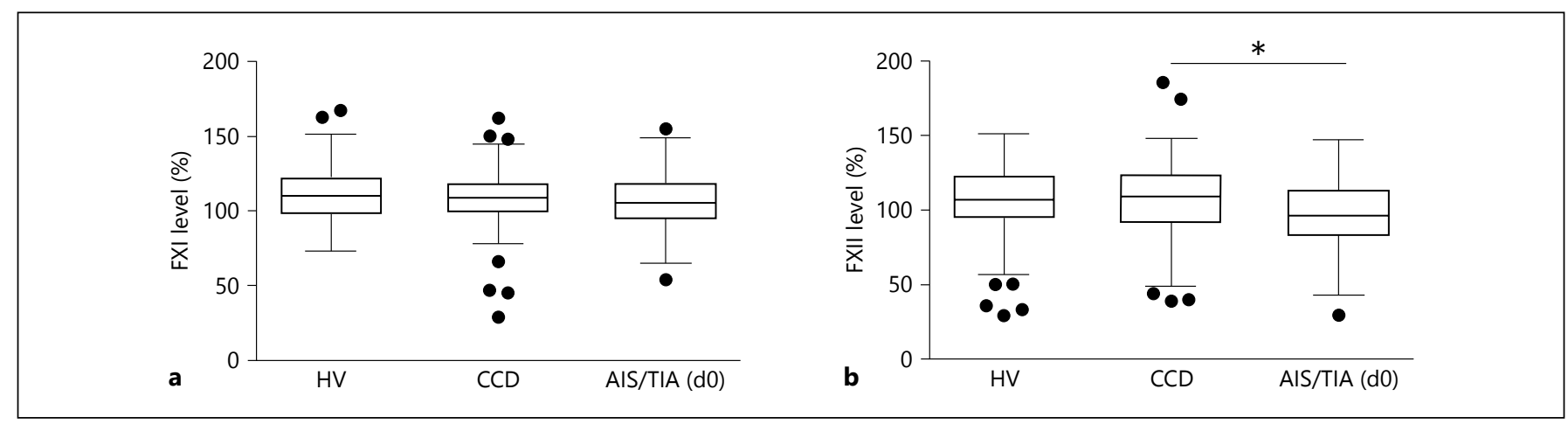

Fig. 1. Factor XI (FXI) and factor XII (FXII) levels in acute ischemic stroke/transitory ischemic attack (AIS/TIA), chronic cerebrovascular disease (CCD), and healthy volunteers (HV). The FXI and FXII levels are depicted in box-and-whisker plots indicating the first and third quartiles as well as the 1.5 interquartile range (IQR, Tukey

$26 \%, \mathrm{p}<0.05$ ) groups (fig. 1). After multivariate adjustment for age and sex, the difference in FXII levels was no longer significant ( $\mathrm{p}=0.552$; data not shown).

\section{Relationship between FXI and FXII Levels and Key}

Demographic and Clinical Parameters in Patients

with an Acute Cerebrovascular Event

FXI levels were significantly correlated with different leukocyte subsets (leukocyte count: $\mathrm{r}=0.21, \mathrm{p}<0.05$; neutrophil count: $r=0.19, p<0.05)$. In contrast, FXII serum levels showed no significant correlation $(\mathrm{p}>0.1)$. Neither FXI nor FXII levels correlated with CRP $(p>0.2)$ (data not shown).

Results from univariate analyses of the association between FXI and FXII levels and key demographic and clinical characteristics are shown in table 2 . Only treatment modality (thrombolysis vs. no thrombolysis) showed a significant association with serum FXI levels $(\mathrm{p}<0.05)$. Age and disease modality (AIS or TIA) demonstrated a trend $(p=0.06)$ for association with higher FXI levels in AIS compared with TIA patients. Evaluation of FXII levels revealed that only age was a significantly associated factor $(p<0.05)$. FXI and FXII levels decreased with increasing patient age and were not associated with clinical severity of stroke (NIHSS, Barthel Index). Sex and the intake of platelet inhibitors had no influence on FXI and FXII levels in the univariate analysis.

In the multivariate analyses, age ( $\mathrm{p}<0.001$ for FXI levels; $\mathrm{p}<0.002$ for FXII levels) and sex ( $\mathrm{p}<0.05$ for FXI levels; $\mathrm{p}=0.05$ for FXII levels) were identified as independent predictors of FXI and FXII levels, respectively (table 3). Treatment and disease modality showed no sig- plot). Outliers outside the $1.5 \mathrm{IQR}$ are visualized by single dots. a FXI levels showed no differences between the three groups. b AIS/ TIA patients had significantly decreased FXII levels compared with CCD patients. Analysis of variance with Bonferroni post-hoc test without adjustment for age and sex. ${ }^{*} \mathrm{p}<0.05$. d0 = day 0 .

nificant influence on biomarker levels in the multivariate analysis. The time point of blood withdrawal (days 0,1 , and 3 ) did not influence FXI and FXII levels ( $p=0.65$ for FXI, $\mathrm{p}=0.19$ for FXII) (data not shown).

\section{Discussion}

In this case-control study, FXII but not FXI serum levels were significantly decreased in acute disorders compared with chronic cerebrovascular disorders. However, differences were no longer significant after adjustment for age and sex. Several demographic and clinical characteristics were found to be associated with FXI and FXII levels.

Several publications showed that FXI and FXII are risk factors for IS [17-20] and are associated with poor functional outcome after stroke [15] and overall mortality [21]. However, other studies found no such association (Review in [30]). Therefore, our findings should be interpreted with caution. Although no independent effect of FXI and/or FXII levels on AIS/TIA risk was found in the multivariate analyses, it cannot be concluded that these factors do not contribute to stroke pathophysiology. We found no significant change in FXI and FXII levels up to day 3 after the incidence of stroke, indicating a persistent rather than an acute modulation of coagulation parameters in response to the AIS/TIA.

Among earlier case-control studies, there have been some inconsistencies with respect to reported results for FXI and FXII levels in patients with IS [17,31]. Additionally, no study up to now has investigated the potential association between etiologic subtypes of IS and FXI or FXII 
Table 2. Predictors of FXI and FXII levels in acute ischemic stroke (AIS)/transitory ischemic attack (TIA) patients by univariate analysis

\begin{tabular}{|c|c|c|c|c|}
\hline & $\begin{array}{l}\text { FXI } \\
(\%)\end{array}$ & $\begin{array}{l}\mathrm{p} \\
\text { value }\end{array}$ & $\begin{array}{l}\text { FXII } \\
(\%)\end{array}$ & $\begin{array}{l}\mathrm{p} \\
\text { value }\end{array}$ \\
\hline \multicolumn{5}{|l|}{ Sex, $n$} \\
\hline Male & $104 \pm 19$ & & $95 \pm 23$ & \\
\hline Female & $110 \pm 27$ & 0.16 & $100 \pm 24$ & 0.29 \\
\hline \multicolumn{5}{|l|}{ Age, years } \\
\hline$<55$ & $114 \pm 19$ & & $113 \pm 28$ & \\
\hline $55-64$ & $115 \pm 26$ & & $100 \pm 24$ & \\
\hline $65-74$ & $108 \pm 23$ & & $98 \pm 20$ & \\
\hline $75-84$ & $100 \pm 20$ & & $90 \pm 21$ & \\
\hline$>84$ & $98 \pm 23$ & 0.06 & $87 \pm 27$ & 0.02 \\
\hline \multicolumn{5}{|l|}{ Disease modality } \\
\hline AIS & $111 \pm 26$ & & $98 \pm 25$ & \\
\hline TIA & $102 \pm 17$ & 0.06 & $96 \pm 22$ & 0.72 \\
\hline \multicolumn{5}{|l|}{ Modified TOAST criteria } \\
\hline Cardioembolism & $107 \pm 23$ & & $96 \pm 24$ & \\
\hline Large-artery atherosclerosis & $110 \pm 24$ & & $109 \pm 19$ & \\
\hline Small-vessel occlusion & $119 \pm 32$ & & $97 \pm 29$ & \\
\hline \multicolumn{5}{|l|}{ Other determined or } \\
\hline determined etiology & $103 \pm 18$ & 0.25 & $98 \pm 23$ & 0.75 \\
\hline \multicolumn{5}{|c|}{ Duration between symptom onset and blood withdrawal, $h$} \\
\hline$<5$ & $111 \pm 18$ & & $103 \pm 24$ & \\
\hline $5-12$ & $99 \pm 21$ & & $95 \pm 24$ & \\
\hline $12-24$ & $107 \pm 25$ & 0.37 & $96 \pm 23$ & 0.30 \\
\hline \multicolumn{5}{|l|}{ National Institutes of Health } \\
\hline \multicolumn{5}{|l|}{ Stroke Scale } \\
\hline $0-4$ & $105 \pm 22$ & & $96 \pm 23$ & \\
\hline $5-9$ & $110 \pm 19$ & & $103 \pm 23$ & \\
\hline $10-15$ & $121 \pm 34$ & & $105 \pm 29$ & \\
\hline$>15$ & $105 \pm 25$ & 0.18 & $85 \pm 27$ & 0.18 \\
\hline \multicolumn{5}{|l|}{ Barthel Index } \\
\hline $0-30$ & $114 \pm 35$ & & $89 \pm 27$ & \\
\hline $35-70$ & $109 \pm 19$ & & $100 \pm 28$ & \\
\hline$>70$ & $107 \pm 19$ & 0.59 & $103 \pm 19$ & 0.21 \\
\hline \multicolumn{5}{|l|}{ Thrombolysis } \\
\hline Yes & $114 \pm 26$ & & $103 \pm 27$ & \\
\hline No & $104 \pm 21$ & 0.03 & $95 \pm 22$ & 0.11 \\
\hline \multicolumn{5}{|c|}{ Platelet inhibitor before blood withdrawal } \\
\hline Yes & $106 \pm 22$ & & $95 \pm 24$ & \\
\hline No & $109 \pm 25$ & 0.62 & $101 \pm 24$ & 0.20 \\
\hline
\end{tabular}

TOAST $=$ Trial of Org 10172 in Acute Stroke Treatment.

serum levels. In fact, our results do not support a major role for stroke etiology in FXI and FXII regulation. However, the relationship between FXI and total leukocyte count and neutrophils in the acute phase after IS noted in our study findings support a role of components of the intrinsic coagulation system in inflammatory processes, especially in thrombo-inflammation in IS $[10,32]$. Thrombo-inflam- mation has been recognized as an important mediator of stroke occurrence and propagation in rodents, and is defined as the interaction of thrombotic (e.g., coagulation factors, platelets) and inflammatory (e.g., immune cells) processes operating at the ischemic neurovascular unit $[10,32]$.

To the best of our knowledge, FXI and FXII levels have not been assessed in a standardized manner in patients with CCD. The observation of unchanged FXI and FXII levels in the AIS/TIA group compared with HV does not rule out a pathophysiologic role for these coagulation factors. Indeed, a recent experimental study showed that FXII regulates the pathologic process of thrombus formation on ruptured plaques [26].

Importantly, growing evidence supports the possibility that clinical as well as therapeutic parameters influence FXI and FXII regulation, which might also have impacted on the FXI and FXII levels measured in our study population [22-25]. Nevertheless, the relevance of such variables on FXI and FXII regulation in different disease settings is unclear. Our findings, and those of other researchers, underline the complexity of associations with FXI and FXII levels. It is well established that estrogen levels and consequently gender [33,34], age [23], as well as genetic polymorphisms [22] influence transcription levels of coagulation proteins such as FXI and FXII. Even if FXI and FXII levels would be suitable as relevant biomarkers of AIS/ TIA, levels of these coagulation factors are influenced by other variables, which confound interpretation of study findings. In contrast to the analysis of von Willebrand factor (VWF) levels in the same study population [35], stroke severity (NIHSS), platelet inhibitor intake before blood withdrawal, and CRP levels were not significantly associated with FXII or FXI levels. Importantly, modality of treatment in the acute phase (i.e., thrombolysis vs. no thrombolysis) was associated with increased FXI and FXII levels in univariate but not in multivariate analysis.

Our findings suggest that the identification of those patients at risk of a cerebrovascular event on the basis of FXI and FXII levels is unlikely to be achievable, thereby indicating that FXI and FXII levels may not be useful biomarkers for that purpose. Accordingly, the proactive and routine screening of FXI and FXII levels to assess the risk of thromboembolic disease in individual patients cannot be recommended at present. Even if FXI and FXII levels could be distinguished between patients with acute and chronic cerebrovascular disease, the current lack of understanding of factors that influence FXI and FXII levels is likely to confound measurements and therefore compromise the interpretation of study findings and the corresponding prediction of cerebrovascular risk. 
Table 3. Predictors of FXI and FXII levels in acute ischemic stroke (AIS)/transitory ischemic attack (TIA) patients by multivariate analysis

\begin{tabular}{|c|c|c|c|c|c|c|}
\hline & \multicolumn{3}{|l|}{ FXI } & \multicolumn{3}{|l|}{ FXII } \\
\hline Male & Reference & & & Reference & & \\
\hline Female & $9.82 \pm 4.40$ & 1.09 to 18.56 & 0.03 & $8.98 \pm 4.59$ & -0.13 to 18.08 & 0.05 \\
\hline Age, years & & & 0.001 & & & 0.002 \\
\hline $65-74$ & $-8.86 \pm 7.05$ & -22.86 to 5.13 & & $-17.41 \pm 7.35$ & -32.00 to -2.82 & \\
\hline $75-84$ & $-16.15 \pm 7.46$ & -30.95 to -1.35 & & $-23.24 \pm 7.77$ & -38.66 to -7.81 & \\
\hline$>84$ & $-21.37 \pm 9.46$ & -40.14 to -2.59 & & $-26.36 \pm 9.86$ & -45.93 to -6.80 & \\
\hline Disease modality (TIA vs. AIS) & $5.50 \pm 4.65$ & -3.72 to 14.71 & & $1.63 \pm 4.84$ & -7.98 to 11.24 & \\
\hline National Institutes of Health Stroke Scale & & & 0.79 & & & 0.72 \\
\hline Thrombolysis & $6.42 \pm 5.68$ & -4.84 to 17.68 & 0.26 & $4.81 \pm 5.92$ & -6.92 to 16.55 & 0.42 \\
\hline \multicolumn{7}{|l|}{ Use of platelet inhibitor before } \\
\hline blood taking & $1.90 \pm 4.64$ & -7.30 to 11.09 & 0.68 & $-1.34 \pm 4.83$ & -10.92 to 8.25 & 0.78 \\
\hline C-reactive protein at admission, $\mathrm{mg} / \mathrm{dl}$ & $0.38 \pm 0.53$ & -0.67 to 1.43 & 0.48 & $-0.12 \pm 0.55$ & -1.21 to 0.97 & 0.83 \\
\hline
\end{tabular}

Our study has several limitations. Firstly, the potential for reverse causation as a result of blood withdrawal post-cerebrovascular event cannot be discounted within the setting of a case-control study. Therefore, it should be emphasized that this study describes the degree and significance of associations between FXI and FXII levels and demographic/clinical parameters, but does not assign causality. Prospective studies are warranted to address this limitation. As ethical guidelines prevented recruitment of patients who were unable to provide informed consent, patients with very large strokes and/or aphasia may not be fully represented in our study. Finally, a non-vascular origin for symptoms could not be fully excluded in a significant proportion of the TIA patient population ( $42 \%)$, and $7 \%$ of patients with AIS/TIA received anticoagulant treatment, which raises the possibility that these factors may have influenced FXI and FXII regulation.

\section{Conclusions}

In this case-control study of patients with acute and chronic cerebrovascular diseases, we have demonstrated that - in contrast to VWF [35] - there is no differential regulation of FXI and FXII: coagulation factors that are increasingly being considered potential biomarkers and promising targets for future therapeutic approaches. Our results show that identification of those patients at risk of a cerebrovascular event, on the basis of FXI and FXII levels, is unlikely to be achievable. However, this study adds to our understanding of the factors that can influence FXI and FXII levels in a given patient.

\section{Acknowledgments}

We thank Melanie Glaser and Andrea Sauer for their excellent technical assistance.

\section{Disclosure Statement}

This publication was funded by the German Research Foundation (Deutsche Forschungsgemeinschaft, Sonderforschungsbereich 688) and the German Ministry for Research and Education (Bundesministerium für Bildung und Forschung, Comprehensive Heart Failure Center, Würzburg, Germany). The funders had no role in study design, data collection and analysis, decision to publish, or preparation of the manuscript. 


\section{References}

1 Stegner D, Nieswandt B: Platelet receptor signaling in thrombus formation. J Mol Med (Berl) 2011;89:109-121.

-2 Kraft P, De Meyer SF, Kleinschnitz C: Nextgeneration antithrombotics in ischemic stroke: preclinical perspective on 'bleedingfree antithrombosis'. J Cereb Blood Flow Metab 2012;32:1831-1840.

-3 Stoll G, Kleinschnitz C, Nieswandt B: Molecular mechanisms of thrombus formation in ischemic stroke: novel insights and targets for treatment. Blood 2008;112:3555-3562.

4 Kleinschnitz C, Stoll G, Bendszus M, Schuh K, Pauer HU, Burfeind P, Renné C, Gailani D, Nieswandt B, Renné T: Targeting coagulation factor XII provides protection from pathological thrombosis in cerebral ischemia without interfering with hemostasis. J Exp Med 2006; 203:513-518.

5 Hagedorn I, Schmidbauer S, Pleines I, Kleinschnitz C, Kronthaler U, Stoll G, Dickneite G, Nieswandt B: Factor XIIa inhibitor recombinant human albumin Infestin-4 abolishes occlusive arterial thrombus formation without affecting bleeding. Circulation 2010;121: 1510-1517.

-6 Macfarlane RG: An enzyme cascade in the blood clotting mechanism, and its function as a biochemical amplifier. Nature 1964;202: 498-499.

7 Kannemeier C, Shibamiya A, Nakazawa F, Trusheim H, Ruppert C, Markart P, Song Y, Tzima E, Kennerknecht E, Niepmann M, von Bruehl ML, Sedding D, Massberg S, Gunther A, Engelmann B, Preissner KT: Extracellular RNA constitutes a natural procoagulant cofactor in blood coagulation. Proc Natl Acad Sci U S A 2007;104:6388-6393.

-8 Müller F, Mutch NJ, Schenk WA, Smith SA, Esterl L, Spronk HM, Schmidbauer S, Gahl WA, Morrissey JH, Renné T: Platelet polyphosphates are proinflammatory and procoagulant mediators in vivo. Cell 2009;139:1143-1156.

$\checkmark 9$ Albert-Weißenberger C, Sirén AL, Kleinschnitz C: Ischemic stroke and traumatic brain injury: the role of the kallikrein-kinin system. Prog Neurobiol 2013;101-102:65-82.

10 Nieswandt B, Kleinschnitz C, Stoll G: Ischaemic stroke: a thrombo-inflammatory disease? J Physiol 2011;589:4115-4123.

-11 Toomey JR, Valocik RE, Koster PF, Gabriel MA, McVey M, Hart TK, Ohlstein EH, Parsons AA, Barone FC: Inhibition of factor IX(a) is protective in a rat model of thromboembolic stroke. Stroke 2002;33:578-585.

- 12 Choudhri TF, Hoh BL, Prestigiacomo CJ, Huang J, Kim LJ, Schmidt AM, Kisiel W, Connolly ES Jr, Pinsky DJ: Targeted inhibition of intrinsic coagulation limits cerebral injury in stroke without increasing intracerebral hemorrhage. J Exp Med 1999;190:91-99.
13 Leung PY, Hurst S, Berny-Lang MA, Verbout NG, Gailani D, Tucker EI, Wang RK, McCarty OJ, Gruber A: Inhibition of factor XII-mediated activation of factor XI provides protection against experimental acute ischemic stroke in mice. Transl Stroke Res 2012;3:381389.

14 Chen JW, Figueiredo JL, Wojtkiewicz GR, Siegel C, Iwamoto Y, Kim DE, Nolte MW, Dickneite G, Weissleder R, Nahrendorf M: Selective factor XIIa inhibition attenuates silent brain ischemia: application of molecular imaging targeting coagulation pathway. JACC Cardiovasc Imaging 2012;5:1127-1138.

15 Undas A, Slowik A, Gissel M, Mann KG, Butenas S: Circulating activated factor XI and active tissue factor as predictors of worse prognosis in patients following ischemic cerebrovascular events. Thromb Res 2011; 128:e62-e66.

16 Siegerink B, Rosendaal FR, Algra A: Antigen levels of coagulation factor XII, coagulation factor XI and prekallikrein, and the risk of myocardial infarction and ischemic stroke in young women. J Thromb Haemost 2014;12: 606-613.

17 Suri MF, Yamagishi K, Aleksic N, Hannan PJ, Folsom AR: Novel hemostatic factor levels and risk of ischemic stroke: the Atherosclerosis Risk in Communities (ARIC) Study. Cerebrovasc Dis 2010;29:497-502.

18 Santamaría A, Oliver A, Borrell M, Belvís R, Martí-Fábregas J, Mateo J, Fontcuberta J: Higher risk of ischaemic stroke associated with factor XI levels in dyslipidaemic patients. Int J Clin Pract 2007;61:1819-1823.

19 Siegerink B, Govers-Riemslag JW, Rosendaal FR, Ten Cate H, Algra A: Intrinsic coagulation activation and the risk of arterial thrombosis in young women: results from the Risk of Arterial Thrombosis in relation to Oral contraceptives (RATIO) case-control study. Circulation 2010;122:1854-1861.

20 Girolami A, Gavasso S, Pacquola E, Cabrio L, Lombardi AM, Girolami B: Comparable levels of activity and antigen in factor XII deficiency: a study of 21 homozygotes and 58 heterozygotes. Clin Appl Thromb Hemost 2005; 11:335-338.

21 Endler G, Marsik C, Jilma B, Schickbauer T, Quehenberger P, Mannhalter C: Evidence of a U-shaped association between factor XII activity and overall survival. J Thromb Haemost 2007;5:1143-1148.

22 Bach J, Endler G, Winkelmann BR, Boehm BO, Maerz W, Mannhalter C, Hellstern P: Coagulation factor XII (FXII) activity, activated FXII, distribution of FXII C46T gene polymorphism and coronary risk. J Thromb Haemost 2008;6:291-296.

-23 Attard C, van der Straaten T, Karlaftis V, Monagle P, Ignjatovic V: Developmental hemostasis: age-specific differences in the levels of hemostatic proteins. J Thromb Haemost 2013;11:1850-1854.
24 Zgraggen L, Fischer JE, Mischler K, Preckel D, Kudielka BM, von Känel R: Relationship between hemoconcentration and blood coagulation responses to acute mental stress. Thromb Res 2005;115:175-183.

25 Wendel HP, Jones DW, Gallimore MJ: FXII levels, FXIIa-like activities and kallikrein activities in normal subjects and patients undergoing cardiac surgery. Immunopharmacology 1999;45:141-144.

26 Kuijpers MJ, van der Meijden PE, Feijge MA, Mattheij NJ, May F, Govers-Riemslag J, Meijers JC, Heemskerk JW, Renné T, Cosemans JM: Factor XII regulates the pathological process of thrombus formation on ruptured plaques. Arterioscler Thromb Vasc Biol 2014; 34:1674-1680.

27 Adams HP Jr, Bendixen BH, Kappelle LJ, Biller J, Love BB, Gordon DL, Marsh EE 3rd: Classification of subtype of acute ischemic stroke. Definitions for use in a multicenter clinical trial. TOAST. Trial of Org 10172 in Acute Stroke Treatment. Stroke 1993;24:3541.

$\checkmark 28$ Muir KW, Weir CJ, Murray GD, Povey C, Lees KR: Comparison of neurological scales and scoring systems for acute stroke prognosis. Stroke 1996;27:1817-1820.

29 Granger CV, Dewis LS, Peters NC, Sherwood CC, Barrett JE: Stroke rehabilitation: analysis of repeated Barthel index measures. Arch Phys Med Rehabil 1979;60:14-17.

30 Gailani D, Renné T: Intrinsic pathway of coagulation and arterial thrombosis. Arterioscler Thromb Vasc Biol 2007;27:2507-2513.

- 31 Undas A, Slowik A, Gissel M, Mann KG, Butenas S: Active tissue factor and activated factor XI in patients with acute ischemic cerebrovascular events. Eur J Clin Invest 2012;42: 123-129.

32 Stoll G, Kleinschnitz C, Nieswandt B: Combating innate inflammation: a new paradigm for acute treatment of stroke? Ann N Y Acad Sci 2010;1207:149-154.

33 Farsetti A, Misiti S, Citarella F, Felici A, Andreoli M, Fantoni A, Sacchi A, Pontecorvi A: Molecular basis of estrogen regulation of Hageman factor XII gene expression. Endocrinology 1995;136:5076-5083.

34 Cleuren AC, van der Linden IK, de Visser YP, Wagenaar GT, Reitsma PH, van Vlijmen BJ: 17alpha-Ethinylestradiol rapidly alters transcript levels of murine coagulation genes via estrogen receptor alpha. J Thromb Haemost 2010;8:1838-1846.

35 Kraft P, Drechsler C, Gunreben I, Nieswandt B, Stoll G, Heuschmann PU, Kleinschnitz C: Von Willebrand factor regulation in patients with acute and chronic cerebrovascular disease: a pilot, case-control study. PLoS One 2014;9:e99851.
Regulation of FXI and FXII in

Cerebrovascular Disease
Cerebrovasc Dis 2014;38:337-343 DOI: $10.1159 / 000368434$ 